\title{
On some Properties of the Schrauben Functions in Connection with the Magnetic Operators
}

\author{
A. D. Jannussis and P. I. Tsilimigras \\ "Demokritos" Nuclear Research Center, Athens, Greece \\ (Z. Naturforsch. 24 a, 340-344 [1969] ; received 25 February 1967)
}

\begin{abstract}
In this paper we introduce two new "magnetic" operators and we show that these are the creation and annihilation operators of the schrauben functions. The schrauben functions are the eigenfunctions of the Hamilton operator describing a free electron under the action of a uniform magnetic field. By transforming these two operators we obtain two other operators which we show to be of Harpers type, as was expected.
\end{abstract}

\section{Introduction}

For the study of special functions occuring in mathematical physics several methods have been used, especially their integral expressions and techniques from the theory of analytic functions.

Recently KaUfMAN $^{1}$ applied the Lie algebra (or Lie group) for the study of the special functions. By this way the addition theorems are obtained in an extremely simple way: many of the expansion theorems are then derived from the addition theorems.

Each Lie group is characterized by an infinitesimal transformation ${ }^{2}$. The basic idea of the Lie group is that from an infinitesimal operator $M$, which shifts the point $S \equiv(x, y, \ldots)$ to a neighboring point one may generate a finite operator $\exp a M$ which shifts the point $S$ into a point $S^{\prime}$ at a finite distance along the path curve of the oneparameter group exp $a M$.

The application of the same operator to a function of the coordinates $F(S)$ yields:

$$
\exp a M F(S)=F\left(S^{\prime}\right) \equiv F(\exp a M S) .
$$

According to KaUfman we consider those infinitesimal differential operators which appear in the recursion relations for the various special functions, and generate from them finite operators. Any pair of recursion relations for the special functions may be rewritten in the form

$$
\begin{aligned}
& R F_{n}=P_{n} F_{n+1} \\
& L F_{n}=\lambda_{n} F_{n-1}
\end{aligned}
$$

where $R$ and $L$ are differential operators and are called raising (creation) and lowering (annihilation)

\footnotetext{
1 B. Kaufman, J. Math. Physics 7, 447 [1966].
}

operators for the index $n$ respectively and $P_{n}, \lambda_{n}$ are constants.

From the relations (2) one easily obtains

$$
\begin{aligned}
& R^{m} F_{n}=P_{n} P_{n+1} \ldots P_{n+m-1} F_{n+m}, \\
& L^{m} F_{n}=\lambda_{n} \lambda_{n-1} \ldots \lambda_{n-m+1} F_{n-m} .
\end{aligned}
$$

The application of the operators $\exp a R, \exp a L$ to the functions $F_{n}(S)$ yields:

$$
\begin{array}{r}
\exp a R \cdot F_{n}(S)=\sum_{m=0}^{\infty} \frac{a^{m}}{m !}\left(P_{n} P_{n+1} \ldots P_{n+m-1}\right) \\
\cdot F_{n+m}(S),
\end{array}
$$

$\exp a L \cdot F_{n}(S)=\sum_{m=0}^{\infty} \frac{a^{m}}{m !}\left(\lambda_{n} \lambda_{n-1} \ldots \lambda_{n-m+1}\right)$
$\cdot F_{n-m}(S)$

These series may or may not terminate, depending on the particular values of $P_{n}, \lambda_{n}$.

The combination of Eqs. (1) and (3) yields two useful relations, one due to the operator $R$ :

$$
\begin{aligned}
F_{n}(\exp a R S) & =F_{n}\left(S^{\prime}\right) \\
& =\sum_{m=0}^{\infty} \frac{a^{m}}{m !} P_{n} P_{n+1} \ldots P_{n+m-1} F_{n+m}(S)
\end{aligned}
$$

and a similar one due to the operator $L$

$$
\begin{aligned}
F_{n}(\exp a L S) & =F_{n}\left(S^{\prime \prime}\right) \\
& =\sum_{m=0}^{\infty} \frac{a^{m}}{m !} \lambda_{n} \lambda_{n-1} \ldots \lambda_{n-m+1} F_{n-m}(S)
\end{aligned}
$$

Further, we can try to combine $R$ and $L$ e.g. in the form $\exp (a R+b L)$. Now, if $R$ and $L$ commute i. e.

we have

$$
[R, L]=R L-L R=0
$$

$$
\exp a R \cdot \exp b L=\exp (a R+b L)
$$

When $[R, L] \neq 0$ Eqs. (7) does not hold.

\footnotetext{
2 H. Rampacher, H. Stumpf, and F. Wagner, Fortschritte der Physik 13, 385 [1965].
} 
The set of such operators which is closed under commutation constitutes the Lie algebra generated by $R, L$. Any member $M$ of the algebra generates a finite operator $\exp a M$; the products of these generate the Lie group corresponding to this Lie algebra.

KaUfMaN ${ }^{1}$ applied the above theory to the special functions and especially to the Bessel functions, Hermite polynomials, Gegenbauer polynomials and Legendre polynomials.

For the study of Bessel functions the appropriate raising and lowering operators are (expressed in polar coordinates) :

$$
\begin{gathered}
R=e^{i \Phi}\left(-\frac{i}{r} \frac{\partial}{\partial \varphi}-\frac{\partial}{\partial r}\right), \\
L=e^{-i \Phi}\left(-\frac{i}{r} \frac{\partial}{\partial \varphi}+\frac{\partial}{\partial r}\right) .
\end{gathered}
$$

From the above definition we easily obtain the relations:

$$
\begin{aligned}
& R\left[e^{i n \varphi} J_{n}(r)\right]=e^{(n+1) i \varphi} \cdot J_{n+1}(r) \\
& L\left[e^{i n \varphi} J_{n}(r)\right]=e^{(n-1) i \varphi} \cdot J_{n-1}(r)
\end{aligned}
$$

where $J_{n}(r)$ are the Bessel functions which the following differential equation fulfil.

$$
\left[\frac{\mathrm{d}^{2}}{\mathrm{~d} r^{2}}+\frac{1}{r} \frac{\mathrm{d}}{\mathrm{d} r}-\frac{n^{2}}{r^{2}}+1\right] J_{n}(r)=0 .
$$

Expressed in Cartesian coordinates the operators (8) become

$$
R=-\frac{\partial}{\partial x}-i \frac{\partial}{\partial y}, \quad L=\frac{\partial}{\partial x}-i \frac{\partial}{\partial y} .
$$

In this paper we define two new operators $\tilde{R}$ and $\tilde{L}$, more general than operator (8), by the relations

$\widetilde{R}=\frac{\partial}{\partial x}-i \frac{\partial}{\partial y}+\frac{B}{2}(x+i y)=-R+\frac{B}{2}(x+i y)$,

$\widetilde{L}=\frac{\partial}{\partial x}+i \frac{\partial}{\partial y}-\frac{B}{2}(x-i y)=L-\frac{B}{2}(x-i y)$.

It will be shown later that the operators $\widetilde{R}$ and $\widetilde{L}$ satisfy Eqs. (2) where $F_{n}(S)$ are the so called Schrauben functions ${ }^{3}$.

\section{Schrauben Functions and their Properties}

It has been shown by JANNUSsis ${ }^{3}$ that for a free electron moving in a homogeneous magnetic field it is more convenient to take the eigenfunctions in

\footnotetext{
3 A. Jannussis, Phys. Stat. Sol. 6, 217 [1964].
}

the form:

$$
\begin{aligned}
& \psi_{\boldsymbol{k}, n}(\boldsymbol{r})=\sqrt{\frac{B}{2 \pi}\left(\frac{2}{B}\right)^{n} \frac{1}{n !}} \\
& \cdot \exp \left(-\frac{1}{B}\left(K_{x}{ }^{2}+K_{y}{ }^{2}\right)+i(\boldsymbol{K}, \boldsymbol{r})\right)\left(-K_{y}-i K_{x}\right)^{n}
\end{aligned}
$$

where

$\boldsymbol{K}$ is the wave vector

$$
K_{x}=k_{x}+\frac{B}{2} y, K_{y}=k_{y}-\frac{B}{2} x, B=\frac{H}{c} .
$$

and $\psi_{\boldsymbol{k}, n}(\boldsymbol{r})$ the schrauben function.

It is assumed here that the magnetic field $H$ is parallel to the $z$-axis and that the vector potential $\boldsymbol{A}(\boldsymbol{r})$ is given by:

$$
\boldsymbol{A}(\boldsymbol{r})=\frac{1}{2}|\boldsymbol{H} \times \boldsymbol{r}| .
$$

All the formulas are referred to atomic units $\hbar=1$, $m=1, e=1$. It can be easily verified that the functions (13) are with centers

$$
\boldsymbol{r}_{m}\left(x_{m}, y_{m}\right): x_{m}=\frac{2}{B} k_{y}, y_{m}=-\frac{2}{B} k_{x}
$$

and the schrauben functions take the following symmetric form:

$$
\begin{aligned}
\psi_{k, n}\left(\boldsymbol{r}, \boldsymbol{r}_{m}\right) & =\sqrt{\frac{B}{2 \pi}\left(\frac{B}{2}\right)^{n} \frac{1}{n !}} \cdot\left[\left(x-x_{m}\right)-i\left(x-y_{m}\right)\right]^{n} \\
\cdot & \exp \left\{-\frac{B}{4}\left[\left(x-x_{m}\right)^{2}+\left(y-y_{m}\right)^{2}\right]\right. \\
+ & \left.i \frac{B}{2}\left(x_{m} y-x y_{m}\right)+i k_{z} z\right\} .
\end{aligned}
$$

It has been shown ${ }^{4}$ that in case $r_{m}$ is a point of a plane lattice then the eigenfunctions (17) are the Wannier functions $\alpha_{n}(\boldsymbol{r})$ of a free electron moving in a homogeneous magnetic field.

It is interesting to note that the operators (12) appear in the Dirac equation describing the behaviour of an electron in a homogeneous magnetic field $\boldsymbol{H}$ and when the magnetic field vanishes there remain the operators (11).

It can be shown the following relations:

$$
\begin{aligned}
& \widetilde{R} \psi_{\boldsymbol{k}, n}(\boldsymbol{r})=\sqrt{2 B n} \psi_{\boldsymbol{k}, n-1}(\boldsymbol{r}), \\
& \widetilde{L} \psi_{\boldsymbol{k}, n}(\boldsymbol{r})=-\sqrt{2 B(n+1) \psi_{\boldsymbol{k}, n+1}(\boldsymbol{r}) .}
\end{aligned}
$$

From the above relations and $n=0$ follows:

$$
\begin{aligned}
& \widetilde{R} \psi_{\boldsymbol{k}, 0}(\boldsymbol{r})=0, \\
& \widetilde{L} \psi_{\boldsymbol{k}, 0}(\boldsymbol{r})=-\sqrt{2 B} \psi_{\boldsymbol{k}, 0}(\boldsymbol{r}) .
\end{aligned}
$$

${ }^{4}$ A. Jannussis, Z. Naturforsch. 21 a, 1577 [1966]. 
By applying $(n-1)$ times the operator $\widetilde{L}$ to the second of the above equations we obtain:

$$
\psi^{\prime}, n(\boldsymbol{r})=\frac{(-1)^{n}}{\sqrt{(2 B)^{n} n !}}(\widetilde{L})^{\mathrm{n}} \psi_{\boldsymbol{k}, 0}(\boldsymbol{r}) .
$$

Consequently, we can derive all the eigenfunctions $\psi_{\boldsymbol{k}, n}(\boldsymbol{r})$ be repeated application of the operator $\widetilde{L}$ to the basic one $\psi_{\boldsymbol{k}, 0}(\boldsymbol{r})$.

The eigenvalues corresponding to the eigenfunctions (13) are the Landau ${ }^{6}$ ones

$$
E_{n}=B\left(n+\frac{1}{2}\right)+\frac{1}{2} k_{z}^{2} \text {. }
$$

\section{Some Properties of the Operators $\widetilde{R}$ and $\widetilde{L}$}

Using the definition (12) of the operators $\widetilde{R}$ and $\widetilde{L}$ we obtain:

$$
\begin{aligned}
\widetilde{R} \widetilde{L}=-R L & +\frac{B}{2} R(x-i y) \\
& +\frac{B}{2}(x+i y) L-\frac{B^{2}}{4}\left(x^{2}+y^{2}\right), \\
\widetilde{L} \widetilde{R}=-L R & +\frac{B}{2} L(x+i y) \\
& +\frac{B}{2}(x-i y) R-\frac{B^{2}}{4}\left(x^{2}+y^{2}\right) .
\end{aligned}
$$

Subtracting the above equations and taking into account that the operators $R$ and $L$ commute we obtain:

$$
\widetilde{R} \widetilde{L}-\widetilde{L} \widetilde{R}=-2 B .
$$

Furthermore, from Eq. (22) we obtain:

$\widetilde{R} \widetilde{L}=\frac{\partial^{2}}{\partial x^{2}}+\frac{\partial^{2}}{\partial y^{2}}+i B\left(y \frac{\partial}{\partial x}-x \frac{\partial}{\partial y}\right)-\frac{B^{2}}{4}\left(x^{2}+y^{2}\right)-B$

$\widetilde{L} \widetilde{R}=\frac{\partial^{2}}{\partial x^{2}}+\frac{\partial^{2}}{\partial y^{2}}+i B\left(y \frac{\partial}{\partial x}-x \frac{\partial}{\partial y}\right)-\frac{B^{2}}{4}\left(x^{2}+y^{2}\right)+B$

The Hamilton operator for a two dimensional motion of an electron in a magnetic field is:

$H_{0}=\frac{1}{2}\left(\frac{\partial^{2}}{\partial x^{2}}+\frac{\partial^{2}}{\partial y^{2}}\right)+i B\left(y \frac{\partial}{\partial x}-x \frac{\partial}{\partial y}\right)-\frac{B^{2}}{4}\left(x^{2}+y^{2}\right)$.

From Eqs. (24) and (25) we obtain:

$$
\begin{aligned}
& \widetilde{R} \widetilde{L}=2 H_{0}-B, \\
& \widetilde{L} \widetilde{R}=2 H_{0}+B .
\end{aligned}
$$

Furthermore, the Schroedinger equation yields:

$H_{0} \psi_{\boldsymbol{k}, n}(\boldsymbol{r})=E_{n} \psi_{\boldsymbol{k}, n}(\boldsymbol{r})=B\left(n+\frac{1}{2}\right) \psi_{\boldsymbol{k}, n}(\boldsymbol{r})$.

5 A. Jannussis, Z. Physik 190, 129 [1965].
From the relations (26) and (27) we obtain

$$
\begin{aligned}
& \widetilde{R} \widetilde{L} \psi_{\boldsymbol{k}, n}(\boldsymbol{r})=2 B n \psi_{\boldsymbol{k}, n}(\boldsymbol{r}), \\
& \widetilde{L} \widetilde{R} \psi_{\boldsymbol{k}, n}(\boldsymbol{r})=2 B(n+1) \psi_{\boldsymbol{k}, n}(\boldsymbol{r}) .
\end{aligned}
$$

From the above results it can easily be shown:

$$
\begin{aligned}
& \widetilde{R} \widetilde{L} \cdot \widetilde{R} \psi_{\boldsymbol{k}, n}(\boldsymbol{r})=2 B(n-1) \widetilde{R} \psi_{\boldsymbol{k}, n}(\boldsymbol{r}), \\
& \widetilde{R} \widetilde{L} \cdot \widetilde{L} \psi_{\boldsymbol{k}, n}(\boldsymbol{r})=2 B(n+1) \widetilde{L} \psi_{\boldsymbol{k}, n}(\boldsymbol{r}) .
\end{aligned}
$$

We conclude now that if $\psi_{\boldsymbol{k}, n}(\boldsymbol{r})$ is an eigenfunction corresponding to the eigenvalue $2 B n$ then $\widetilde{R}_{\psi_{\boldsymbol{k}}, n}(\boldsymbol{r})$ is an eigenfunction corresponding to the eigenvalue $2 B(n-1)$, and $\widetilde{L} \psi_{\boldsymbol{k}},(\boldsymbol{r})$ is an eigenfunction corresponding to the eigenvalue $2 B(n+1)$. Similar relations appear in the study of harmonic oscillators where we have the corresponding creation and annihilation operators ${ }^{2}$.

To find further properties and for the sake of simplicity, we introduce new variables:

$$
\begin{gathered}
\mathcal{U}=x-x_{m}+i\left(y-y_{m}\right), \quad \mathcal{V}=x-x_{m}-i\left(y-y_{m}\right), \\
z_{m}=x_{m}+i y_{m}, \quad z_{m}^{*}=x_{m}-i y_{m} .
\end{gathered}
$$

Eq. (17) and the operators (12) take now the following form:

$$
\begin{aligned}
& \psi_{n}(\mathcal{U}, \mathcal{V})=\sqrt{\frac{B}{2 \pi}\left(\frac{B}{2}\right)^{n} \frac{1}{n !}} \\
& \mathcal{V}^{n} \exp \left[-\frac{B}{4}\left(\mathcal{U} \mathcal{V}-\mathcal{U} z_{m}^{*}+\mathcal{V} z_{m}\right),\right] \\
& \widetilde{R}=2 \frac{\partial}{\partial \mathcal{V}}+\frac{B}{2}\left(\mathcal{V}+z_{m}\right), \quad \widetilde{L}=2 \frac{\partial}{\partial \mathcal{U}}-\frac{B}{2}\left(\mathcal{V}+z_{m}^{*}\right) .
\end{aligned}
$$

The application of the operator $\exp a L$ to the function $\psi_{n}(\mathcal{U}, \mathcal{V})$ yields:

$\exp a L \cdot \psi_{n}(\mathcal{U}, \mathcal{V})=\sum_{m=0}^{\infty} \frac{a^{m}}{m !} P_{n} \ldots P_{n+m-1} \psi_{n+m}(\mathcal{U}, \mathcal{V})$ or

$\exp a L \cdot \psi_{n}(\mathcal{U}, \mathcal{V})=\exp \{-\alpha B \mathcal{V}\} \psi_{n}(\mathcal{U}, \mathcal{V})$.

Eq. (33) can be verified and by the following procedure:

$$
\begin{gathered}
\exp a L \cdot \psi_{n}(\mathcal{U}, \mathcal{V})=\exp \left\{2 a \frac{\partial}{\partial \mathcal{U}}-\frac{a B}{2}\left(\mathcal{V}+z^{*}\right)\right\} \\
\psi_{n}(\mathcal{U}, \mathcal{V})=\exp \left[-\frac{a B}{2}\left(\mathcal{V}+z^{*}\right)\right] \\
\cdot \psi_{n}(\mathcal{U}+2 a, \mathcal{V})=\exp \{-a B \mathcal{V}\} \cdot \psi_{n}(\mathcal{U}, \mathcal{V}) .
\end{gathered}
$$

Proceeding in a similar way we have

$$
\exp a \widetilde{R} \cdot \psi_{n}(\mathcal{U}, \mathcal{V})=\left(1+\frac{2 a}{\mathcal{V}}\right)^{n} \cdot \psi_{n}(\mathcal{U}, \mathcal{V}) \text {. }
$$

${ }^{6}$ L. Landau, Z. Physik 64, 629 [1930]. 
We define now two new operators $L_{0}$ and $R_{0}$ as follows:

$$
\begin{aligned}
& L_{0}=\frac{1}{2}(\tilde{L}+\widetilde{R})=\frac{\partial}{\partial x}+i \frac{B}{2} y, \\
& R_{0}=\frac{i}{2}(\widetilde{L}-\widetilde{R})=\frac{\partial}{\partial y}-i \frac{B}{2} y .
\end{aligned}
$$

These two operators satisfy the following relations

$$
\begin{gathered}
{\left[L_{0}, R_{0}\right]=i B,} \\
L_{0} \psi_{n}\left(x, y, x_{m}, y_{m}\right) \quad \sqrt{\frac{B}{2}}\left(\sqrt{n} \psi_{n-1}-\sqrt{n+1} \psi_{n+1}\right), \\
R_{0} \psi_{n}\left(x, y, x_{m}, y_{m}\right) \\
=\sqrt{\frac{B}{2}}\left(\sqrt{n} \psi_{n-1}+\sqrt{n+1} \psi_{n+1}\right) .
\end{gathered}
$$

Consider now the conjugate operators $L_{0}{ }^{*}, R_{0}{ }^{*}$

$$
L_{0}^{*}=\frac{\partial}{\partial x}-i \frac{B}{2} y, \quad R_{0}^{*}=\frac{\partial}{\partial y}+i \frac{B}{2} x .
$$

The following pair of formulas can be obtained:

$\exp a L_{0}{ }^{*} \cdot \psi_{n}\left(x, y, x_{m}, y_{m}\right)=\exp \left\{-i \frac{a B}{2} y+a \frac{\partial}{\partial x}\right\}$

$$
\begin{aligned}
& \cdot \psi_{n}\left(x, y, x_{m}, y_{m}\right)=\exp \left\{-i \frac{a B}{2} y\right\} \\
& \cdot \psi_{n}\left(x, y, x_{m}, y_{m}\right) \\
& \exp a R_{0}^{*} \cdot \psi_{n}\left(x, y, x_{m}, y_{m}\right)=\exp \left\{i \frac{a B}{2} x\right\} \\
& \cdot \psi_{m}\left(x, y+a, x_{m}, y_{m}\right) .
\end{aligned}
$$

Taking into account that the eigenfunctions (17) satisfy the Harper symmetric condition ${ }^{7}$ we finally obtain:

$$
\begin{gathered}
\exp a L_{0}{ }^{*} \cdot \psi_{n}\left(x, y, x_{m}, y_{m}\right)=\exp \left\{-i \frac{B a}{2} y_{m}\right\} \\
\psi_{n}\left(x, y, x_{m}-a, y_{m}\right), \\
\exp a R_{0}{ }^{*} \cdot \psi_{n}\left(x, y, x_{m}, y_{m}\right)=\exp \left\{\begin{array}{c}
\left.i \frac{B a}{2} x_{m}\right\} \\
\psi_{n}\left(x, y, x_{m}, y_{m}-a\right) .
\end{array}\right.
\end{gathered}
$$

The operators $\exp a L_{0}{ }^{*}$ and $\exp a R_{0}{ }^{*}$ are thus of the HARPER ${ }^{7}$ type.

\section{The Harper Operators and their Properties}

The Hamilton operator of a lattice electron moving in a homogeneous magnetic field does not commute with the translation operator, but HARPER $^{7}$

7 P. Harper, Proc. Phys. Soc. London A 68, 879 [1955].

8 W. Kohn, Phys. Rev. 115, 1460 [1959].

9 L. Roth, J. Phys. Chem. Solids 23, 433 [1962].

10 G. Wannier and D. Fredkin, Phys. Rev. 125, 1910 [1962]. was able to show that there is a set of so called "Eichtranslationen" (or Harper operators) which commute with this Hamilton operator and have the following form:

$$
C\left(\boldsymbol{r}_{0}\right)=\exp \left\{-\frac{i}{c}\left(\boldsymbol{A}\left(\boldsymbol{r}_{0}\right), \boldsymbol{r}\right)\right\} \cdot T \bar{r}_{0} .
$$

$T_{r_{0}}$ is the translation operator

$$
T_{\boldsymbol{r}_{0}} f\left(\boldsymbol{r}_{0}\right)=f\left(\boldsymbol{r}+\boldsymbol{r}_{0}\right)
$$

and $\boldsymbol{A}\left(\boldsymbol{r}_{0}\right)$ is the vector potential at the point $\boldsymbol{r}_{0}$.

From the definition (40) follows at once

$$
\begin{aligned}
& \exp a L_{0}{ }^{*}=C(a, 0,0), \\
& \exp a R_{0}{ }^{*}=C(0, a, 0) .
\end{aligned}
$$

Moreover, we can easily verify the commutation rule

$$
\begin{aligned}
& {\left[C\left(\boldsymbol{r}_{0}\right) C\left(\boldsymbol{r}_{1}\right)-C\left(\boldsymbol{r}_{1}\right) C\left(\boldsymbol{r}_{0}\right)\right]} \\
& \quad=2 i \sin \frac{B}{2}\left(y_{1} x_{0}-x_{1} y_{0}\right) \cdot C\left(\boldsymbol{r}_{0}+\boldsymbol{r}_{1}\right) .
\end{aligned}
$$

In case the vectors $\boldsymbol{r}_{0}, \boldsymbol{r}_{1}$ and the magnetic field are coplanar then the Harper operators form an Abelian group and the relation (43) gives at once

$$
\frac{B}{2}\left(y_{1} x_{0}-x_{1} y_{0}\right)=\pi l .
$$

Formula (44) expresses the quantization of the magnetic field.

It will be shown now that the operators $T_{r^{0}}$ and $\exp \left\{-i / c\left(\boldsymbol{A}\left(\boldsymbol{r}_{0}\right), \boldsymbol{r}\right)\right\}$ commute. Indeed, by using the definition (41) and the fact that $\left(\boldsymbol{A}\left(\boldsymbol{r}_{0}\right), \boldsymbol{r}_{0}\right)=0$ we obtain

$$
\begin{aligned}
& T_{\boldsymbol{r}_{0}} \cdot \exp \left\{-\frac{i}{c}\left(\boldsymbol{A}\left(\boldsymbol{r}_{0}\right), \boldsymbol{r}\right)\right\} \cdot f(\boldsymbol{r}) \\
& =\exp \left\{-\frac{i}{c}\left(\boldsymbol{A}\left(\boldsymbol{r}_{0}\right), \boldsymbol{r}\right)\right\} \cdot T_{\boldsymbol{r}_{0}} f(\boldsymbol{r}) .
\end{aligned}
$$

There now exists an extensive literature devoted to the properties of the Harper operators (see $\mathrm{KOHN}^{8}$, Roth ${ }^{9}$, Wannier-FredKin ${ }^{10}$, Blount ${ }^{11}$, Fish$\mathrm{BECK}^{12}$ and $\left.\mathrm{ZAK}^{13}\right)$.

\section{Generalized Harpers Operators}

We define two new operators $C_{\boldsymbol{k}}\left(\boldsymbol{r}_{0}\right)$ and $\bar{C}_{\boldsymbol{k}}\left(\boldsymbol{r}_{0}\right)$ as follows:

$$
\begin{aligned}
& C_{\boldsymbol{k}}\left(\boldsymbol{r}_{0}\right)=\exp \{-i(\boldsymbol{k}, \boldsymbol{r})\} \cdot T_{\boldsymbol{r}_{0}}, \\
& \bar{C}_{\boldsymbol{k}}\left(\boldsymbol{r}_{0}\right)=T_{\boldsymbol{r}_{0}} \exp \{-i(\boldsymbol{k}, \boldsymbol{r})\} .
\end{aligned}
$$

11 E. Blount, Phys. Rev. 126, 1636 [1962].

12 H. Fishbeck, Phys. Stat. Sol. 3, 1082, 2399 [1963].

13 J. ZAK. Phys. Rev. 139, 1159 [1965]. 
From the above relations, we easily obtain

$$
\overline{C_{k}}\left(\boldsymbol{r}_{0}\right)=\exp \left\{-i\left(\boldsymbol{k}, \boldsymbol{r}_{0}\right)\right\} \cdot C_{\boldsymbol{k}}\left(\boldsymbol{r}_{0}\right) .
$$

In case the scalar product $\left(\boldsymbol{k}, \boldsymbol{r}_{0}\right)$ is an integral multiple of $2 \pi$, then the operators $C_{\boldsymbol{k}}\left(\boldsymbol{r}_{0}\right)$ and $C_{\boldsymbol{k}}\left(\boldsymbol{r}_{\mathbf{0}}\right)$ are equal and hence the operators $\exp \{-i(\boldsymbol{k}, \boldsymbol{r})\}$ and $T r_{0}$ commute.

The scalar product $(\boldsymbol{k}, \boldsymbol{r})$ vanishes when the wave vector $\boldsymbol{k}$ is the inverse of the vector $\boldsymbol{r}_{0}$ or when the $\boldsymbol{k}$ is given by the relation

$$
\boldsymbol{k}=\frac{1}{2}\left|\boldsymbol{H} \times \boldsymbol{r}_{1}\right|=\frac{1}{c} \boldsymbol{A}\left(\boldsymbol{r}_{1}\right)
$$

and the vectors $\boldsymbol{r}_{0}, \boldsymbol{r}_{1}$ and $\boldsymbol{H}$ are coplanar.

In the case $\boldsymbol{r}_{1}=\boldsymbol{r}_{0}$ we obtain the Harper's operator $(40)$.

An analytic study of the condition (49) with the three vectors $\boldsymbol{r}_{0}, \boldsymbol{r}_{1}$ and $\boldsymbol{H}$ coplanar, has been made by FishBeCK ${ }^{12}$ and is just the condition for the Harper's operator to form an Abelian group. It can be shown that the operators (46) and (47) form a group and do not commute.

\title{
Solution of the Dirac Equation for the Rectilinear Periodic Motion of an Electron
}

\author{
A. D. JANNUSSIS \\ "Demokritos" Nuclear Research Center, Athens, Greece \\ (Z. Naturforsch. 24 a, $344-349$ [1969] ; received 25 February 1967)
}

\begin{abstract}
In this paper the Dirac equation for a rectilinear onedimensional periodic potential is treated. It is shown that the energy eigenvalues are periodic functions of the wave number $K_{x}$ and the continuous spectrum is split into energy bands. The end points of the energy bands are the points where the Bragg reflection takes place.

These results are obtained by perturbation theory, as well as by the method of determinants, since the resulting eigenvalue equation has the form of a determinant which is similar to the Hill determinant.
\end{abstract}

\section{Introduction}

We consider the motion of an electron parallel to the $x$-axis in a field that is a function of $x$ only. We suppose that the vector potential $A$ is zero everywhere and that $e \varphi=-V(x)$. The Dirac wave function may be written in the form:

$$
\psi=f(x) \exp \left\{(i / \hbar)\left(P_{2} y+P_{3} z-W t\right)\right\} .
$$

Substituting this expression into the Dirac equation we obtain:

$$
\begin{array}{r}
{\left[-i \hbar \alpha_{1} \frac{\mathrm{d}}{\mathrm{d} x}+\frac{W-V(x)}{c}\right.} \\
\left.+\alpha_{2} P_{2}+\alpha_{3} P_{3}+\alpha_{4} m c\right] f(x)=0
\end{array}
$$

where $\alpha_{i}, i=1,2,3,4$ are the Dirac matrices and they satisfy the relations

$$
\alpha_{i} \alpha_{j}+\alpha_{j} \alpha_{i}=2 \delta_{i j} .
$$

The Eq. (2) in scalar function $f_{i}(x)$ has the form:

1 N. Mott and I. Sneddon, Wave Mechanics and its Applications, \& 56, Clarendon Press, Oxford 1948.

$$
\begin{aligned}
& -i \hbar \frac{\mathrm{d} f_{1}}{\mathrm{~d} x}+\frac{W-V(x)}{c} f_{4}+i P_{2} f_{1}-P_{3} f_{3}-m c f_{4}=0, \\
& -i \hbar \frac{\mathrm{d} f_{2}}{\mathrm{~d} x}+\frac{W-V(x)}{c} f_{3}-i P_{2} f_{2}+P_{3} f_{1}-m c f_{3}=0, \\
& -i \hbar \frac{\mathrm{d} f_{3}}{\mathrm{~d} x}+\frac{W-V(x)}{c} f_{2}+i P_{2} f_{3}-P_{3} f_{4}+m c f_{2}=0, \\
& -i \hbar \frac{\mathrm{d} f_{4}}{\mathrm{~d} x}+\frac{W-V(x)}{c} f_{1}-i P_{2} f_{4}+P_{3} f_{3}+m c f_{1}=0 .
\end{aligned}
$$

If we substitute the expressions ${ }^{1}$

$$
\begin{gathered}
f_{2}=\frac{i P_{3}}{K} \varphi_{2}, \quad f_{1}=\varphi_{1}-\frac{P_{2}+i m c}{K} \varphi_{2}, \\
f_{3}=-\frac{i P_{3}}{K} \varphi_{2}, \quad f_{4}=\varphi_{1}+\frac{P_{2}+i m c}{K} \varphi_{2} \\
\text { where } \quad K^{2}=P_{2}{ }^{2}+P_{3}{ }^{2}+m^{2} c^{2} .
\end{gathered}
$$

We obtain the folowing linear system of differential equations for the functions $\varphi_{1}$ and $\varphi_{2}$

$$
\begin{aligned}
& \frac{\mathrm{d} \varphi_{1}}{\mathrm{~d} x}+\frac{i}{\hbar} \frac{W-V(x)}{c} \varphi_{1}+{ }_{\hbar}^{K} \varphi_{2}=0 \\
& \frac{\mathrm{d} \varphi_{2}}{\mathrm{~d} x}-\frac{i}{\hbar} \frac{W-V(x)}{c} \varphi_{2}+\frac{K}{\hbar} \varphi_{1}=0
\end{aligned}
$$

